\title{
El valle de Mexicali: agricultura e inversión extranjera (1901-1986)
}

\author{
Martha Stamatis Maldonado \\ Instituto de Investigaciones Sociales \\ Universidad Autónoma de Baja California
}

\section{Resumen}

El interés de este trabajo es intentar explicar el desarrollo de la agricultura del valle de Mexicali a través de su relación con la inversión extranjera (norteamericana). Relación que está determinada por la función conformadora del sistema económico internacional representada por el capital norteamericano, la cual se va convirtiendo en un hecho, sobre todo a partir de 1940 en la integración nacional de las actividades económicas y sociales que se desarrollan en México. Este proceso de desarrollo ofrece manifestaciones particulares, la agricultura del valle no está ajena a las determinaciones ejercidas por el capital norteamericano sobre la agricultura nacional. De esta forma, este capital sigue manteniendo su presencia en el valle como parte de la función hegemónica para internacionalizar la agricultura mexicana. Las posibilidades que el valle tiene para un mayor crecimiento estriban en factores que permitirían un mayor desplazamiento de la producción de California, tomando en cuenta la corta distancia, entre otros, para que tuvieran un peso suficiente que las convirtiera en ventajas reales.

Palabras clave: valle de Mexicali, agricultura, inversión extranjera, sistema económico internacional, producción agrícola nacional.

\begin{abstract}
The interest of this article is to explain the development of agriculture at the Valley of Mexicali through its relation to foreign investment (American). This relation is conditioned by the conforming function of the international economical system which is represented by the American capitals. Since the early 40's, this situation has become a visible fact to the national integration of social and economical activities developing in Mexico. This development process has particular expressions, and the agriculture of the valley has known the decisions taken by the American capitals upon national agriculture. In this way, this capital is still present in the valley as part of a hegemonic function to internationalize Mexican agriculture. The possibilities of the valley to grow more, depends on factors that could allow a larger expansion of production from California, such as its closeness, just to mention one, that were too convenient to constitute true advantages.
\end{abstract}

Keywords: Mexicali Valley, agricultura, foreing investment, international economic system, domestic agricultural production. 


\title{
EL VALLE DE MEXICALI: AGRICULTURA E INVERSION EXTRANJERA (1901-1986) *
}

\author{
Por \\ Martha Stamatis Maldonado **
}

\section{INTRODUCCION}

El interés de este trabajo es intentar explicar el desarrollo de la agricultura del valle de Mexicali a través de su relación con la inversión extranjera (norteamericana). Relación que está determinada no por su situación fronteriza con Estados Unidos, sino por la función conformadora del sistema económico internacional representada por el capital norteamericano y sobre todo a partir de 1940, en la integración nacional de las actividades económico sociales que se desarrollan en México se va convirtiendo en un hecho, consideramos que no se puede explicar el desarrollo agrícola del valle sin tomar en cuenta el desarrollo agrícola general del pais.

Existe en la región, la visión tradicional de concebir el desarrollo del valle de Mexicali como cualitativamente diferente al del resto de las zonas agrícolas del país, derivada de su situación fronteriza y de los orígenes mismos del valle; visión que, por lo demás, se ha ido deteriorando a partir de las fuertes devaluaciones y de su consiguiente mayor integración al mercado nacional.

$\mathrm{Y}$, si bien es cierto que csa situación ha matizado ese proceso de desarrollo dándole una gran cantidad de manifestaciones particulares, consideramos que la agricultura del valle no se sustrae a las determinaciones que ha ejercido el capital norteamericano en sus tendencias de participación sobre la agricultura nacional.

Así, el capital norteamericano ha mantenido su presencia en el valle en forma cambiante, de acuerdo con las necesidades que ha tenido en las diferentes etapas de la conformación del sistema económico internacional y adaptándose a las también cambiantes condiciones juridico-políticas y sociales del valle, como parte de la función hegemónica que ha cumplido en la internacionalización de la agricultura mexicana.

\footnotetext{
- Ponencia presentada en el "I Encuentro sobre Problemas del Campo en el Noroeste de México y el Suroeste de Estados Unidos", en enero de 1987, Culiacán, Sin.

*- Investigadora del Instituto de Investigaciones Sociales de la Universidad Autónoma de Baja California.
} 


\section{PERIODO I: 1901-1937/40. INVERSION EXTRANJERA DIRECTA CON UNA MODALIDAD TIPO AGRICULTURA DE PLANTACION}

El valle de Mexicali y el Valle Imperial forman una unidad geofísica dividida por la línea fronteriza con Estados Unidos. Cuando los norteamericanos empiezan a desarrollar la agricultura del Valle Imperial - a fines del siglo pasado - , conciben el valle de Mexicali como una extensión que les interesa anexarse, y adquieren doscientas mil hectáreas con fines de colonización, bajo la denominación social de Colorado River Land Co. Para 1907, la Colorado es propietaria de trescientas cincuenta mil hectáreas - prácticamente todo el valle-y recibe el reconocimiento legal de su propiedad del régimen de Porfirio Díaz a cambio del compromiso de colonizar esas tierras completamente despobladas.

El valle de Mexicali surge en 1901, en el momento en que sus tierras reciben la primera agua de riego ${ }^{1}$ del río Colorado, a través del sistema de canales construido por la Sociedad de Irrigación y Terrenos de la Baja California, filial de la Mexican Land and Colonization Co.

En estos años, la principal forma de inversión del capital extranjero en América Latina se da bajo la forma de agricultura de plantación la cual podemos caracterizar como una forma de inversión extranjera directa, que supone la propiedad de la tierra, propiedad y control de los procesos de producción y comercialización, y una producción destinada al mercado externo.

El valle de Mexicali no se sustrae a esa tendencia general del capital internacional; más bien, su desarrollo agrícola en este período asume las características esenciales de la agricultura de plantación. La Colorado River Land Co., es propietaria de la tierra y destina su uso al cultivo del algodón; la empresa subsidiaria filial de la Mexican Land and Colonization Co. es propietaria del sistema de riego; y otra subsidiaria, la filial de la Anderson Clayton Co. (ACCO), con el nombre de Cía. Jabonera del Pacífico financia y adquiere la producción de algodón.

Así, la propiedad de la tierra y del agua, de otros recursos productivos, del financiamiento y de la producción para su comercialización en el mercado externo, se encuentran completamente en manos del capital norteamericano. No en balde, esas compañias se jactaban de poseer "el rancho algodonero más grande del mundo".

Y, debido a ciertas particularidades, principalmente el despoblamiento de su territorio y la contigüidad geográfica con territorio norteamericano, adopta una modalidad propia: la necesidad que tiene el capital norteamericano para colonizar el territorio, de concesionar las

${ }^{1}$ En el valle de Mexicali no hay condiciones climatológicas para la agricultura de temporal. 
tierras a arrendatarios chinos; ${ }^{2}$ y la facilidad que tiene de, por un lado, introducir tecnología similar a la que usa en su propio territorio y, por otro, de abastecer con bienes consumo norteamericanos a sus trabajadores (arrendatarios y jornaleros).

La Guerra Mundial (1916) y las leyes de inmigración estadounidenses (1917 y 1924) 3 , aunadas a la promulgación de la "ley seca" en Estados Unidos, hicieron de esta zona fronteriza un foco de enorme atracción para los habitantes del interior del país (de Sonora y Sinaloa principalmente). Una parte de la población "atraída" la utilizó como lugar de asentamiento "puente", mientras cruzaban la línea para trabajar en los campos de California; y otra, como asentamiento permanente para emplearse en los establecimientos de comercio y servicios turísticos en la ciudad de Mexicali.

Con la Gran Depresión de y la derogación de la "ley seca" en 1933, muchos mexicanos regresaron al país y se cerraron la mayoría de los establecimientos turísticos. De los así desempleados, una parte se regresó a sus lugares de origen, pero otra permaneció en el valle, quienes se fueron contratando como jornaleros con los arrendatarios chinos y fue surgiendo la inconformidad con la presencia de la Colorado, que les impedía ser propietarios de la tierra y no les permitía siquiera convertirse en arrendatarios.

En 1930 un grupo de trabajadores mexicanos toman tierras de la Colorado, son encarcelados y enviados a las Islas Marías. Pero una nueva toma de tierras en enero de 1937 sí prosperó, gracias a la política cardenista de nacionalización de las propiedades de extranjeros. Este hecho es conocido en el valle como el "asalto a las tierras" y se conmemora el día 27 de enero de cada año.

\footnotetext{
${ }^{2}$ La política de abastecimiento de mano de obra con la importación de chinos, se encontraba muy extendida en el estado de California: en 1986 se empleaban 30 mil chinos en las cosechas. En 1882 "se aprobó la Chinese Exclusion Act, que prohibía la entrada de chinos por 10 años, la Geary Act la prorrogó por otros 10 años, hasta que en 1902 y 1904, el Congreso de Estados Unidos extendió indefinidamente la prohibición, al mismo tiempo que les negaba la posibilidad de naeionalizarse americanos". Esto, impedía que los chinos emigraran a Estados Unidos en busca de mejores condiciones y lo poco atractiva que resultaba para los mexicanos la permanencia en el valle de Mexicali - pues preferían emigrar debido a los mejores ingresos - hizo que la Colorado adoptara una total preferencia para colonizar con chinos. Para 1919 había en el valle de Mexicali más de 5 mil agricultores chinos. Ver: ZAZUETA, Carlos. La formación de la frontera norte: el caso de Baja California, Tesis de Maestría, El Colegio de México, México, 1978.

${ }^{3}$ La ley de 1917, “. . . corta drásticamente el flujo de inmigrantes europeos (. . .). El test de alfabetismo de esa ley no se aplica a inmigrantes mexicanos debido a la presión que ejercen los empresarios agrícolas del Sur de E.U." La ley de 1924 asignaba cuotas de inmigrantes a cada país e incluso excluía a los chinos u otros orientales; ". . . como concesión al agribusiness los mexicanos no tenían cuota, permitiendo su entrada ilimitada". Ibid, p. 47.

- La promulgación de la "ley seca" en E.U. impulsó un crecimiento acelerado y desmedido de actividades como el comercio y los servicios turísticos (restaurantes, cantinas, prostíbulos, etc.) en la ciudad de Mexicali, lo cual atrajo a gran cantidad de mexicanos, si bien eran los chinos los que controlaban esas actividades.
} 


\section{PERIODO II: 1937/40-1967/70. INVERSION EXTRANJERA INDIRECTA VIA CONTROL DE COMERCIALIZACION DE LA PRODUCCION AGRICOLA PARA EL MERCADO MUNDIAL}

En 1937, régimen del Presidente Cárdenas, se expropian las ciento treinta y tres mil hectáreas que la Colorado tenía en explotación y se constituyen cuarenta y cuatro ejidos y colonias agrícolas. Así, casi un siglo después de los Tratados de Guadalupe Hidalgo, se lleva a cabo la colonización del valle con mexicanos.

Con la nacionalización de la tierra - y posteriormente del agua -5 , se da una relativa recuperación de la producción agrícola para el capital nacional; el capital extranjero pierde la propiedad de la tierra y el control directo sobre el proceso de producción. El Estado interviene de manera decidida en materia agraria, al llevar a cabo de manera efectiva el reparto de la tierra.

Aunque el capital extranjero no tiene una presencia masiva en la zona y sólo tiene interés en algunas compañías algodoneras, su presencia resulta significativa, considerando que la actividad económica derivada del cultivo del algodón es, con mucho, la más importante ${ }^{6}$.

Ante las nuevas condiciones jurídico-políticas, el capital norteamericano adopta una nueva forma de participación en la agricultura del valle. Las compañías que despepitan y empacan el algodón se encuentran a cargo de productores nacionales. Estas otorgan créditos a los agricultores algodoneros mediante contratos de compra-venta de la cosecha ${ }^{7}$. A su vez, la ACCO financia parte de las actividades de esas compañías, adquiere su producción y la comercializa en el mercado mundial.

Puede decirse que la agricultura del valle se encuentra, para el año de 1940, previamente sensibilizada a la tecnología moderna, como otras zonas de agricultura comercial del país. ${ }^{8}$ Sin embargo, recibe un mayor impulso como consecuencia de la nueva forma de inversión del capital extranjero en la agricultura en México.

\footnotetext{
${ }^{5}$ A pesar de haberse nacionalizado la tierra, la Compañía de Terrenos y Aguas de la Baja California siguió proporcionando riego y cobrando las cuotas, de la misma manera que a principios de siglo. Es hasta 1944, con el Tratado sobre el Uso de las Aguas Internacionales suscrito entre México y Estados Unidos, que México adquiere, por derecho, la facultad de disponer de las aguas que de hecho le pertenecían, incluso en condiciones sumamente desfavorables. Y, como resultado de los problemas en la distribución de agua que surgen a partir de 1955, en 1964 se aprueba el Reglamento para el funcionamiento del Distrito de Riego ( $\left.\mathrm{N}^{\circ} 14\right)$, en el que se asignan derechos de riego para 20 hectáreas máximo, por productor. Ver: LOPEZ Zamora, Emilio, El Agua, La Tierra, Los Hombres de México. Ed. FCE., México, 1977, Cap. II.

- Con el crecimiento de la producción algodonera, adquiere importancia su aprovechamiento industrial. A fincs de los cincuentas, el $80 \%$ del valor de la producción agrícola y más del $50 \%$ de la producción industrial, corresponden al algodón.

${ }^{2}$ Esos créditos están constituidos en parte, no por dinero en efectivo, sino por insumos y maquinaria distribuidos por subsidiarias de la ACCO.

- La Comarca Lagunera, los valles del Fuerte y de Culiacán en Sinaloa.
} 
En este período, la principal forma de participación del capital norteamericano sobre la agricultura mexicana se da vía la introducción de "paquetes tecnológicos", que incluyen semilla mejorada e insumos de alto rendimiento para ser utilizados en conjunto, en tierras que permitan el control del riego y la introducción de maquinaria y equipo agrícola que, como un trasplante de la tecnología norteamericana, se utilizan en zonas de agricultura comercial. Todo esto hizo posible el crecimiento acelerado de la producción y la productividad, generándose los más altos volúmenes de producción y exportación registrados en la historia de la agricultura en México.

$Y$, al igual que para la producción agrícola de México, las coyunturas de las guerras - Segunda Guerra Mundial y Guerra de Corea - propician condiciones favorables para la producción algodonera del valle. El crecimiento de la demanda y el alza de los precios internacionales del algodón, provocaron un acendramiento tal en la especialización de la agricultura del valle que, aunado al régimen de zona libre, ${ }^{9}$ generaron un sistema de monocultivo en exceso.

En este sentido, la situación fronteriza y el régimen de zona libre le dan evidentes particularidades a la agricultura del valle. El no formar parte de un mercado interno "cautivo" y tener la facilidad de depender del abastecimiento de bienes de consumo del mercado norteamericano, obstaculiza una mayor integración a la economía nacional y un cierto grado de diversificación de la oferta agrícola.

En cuanto al abastecimiento de insumos a la producción agrícola, aunque existen ciertas facilidades arancelarias, esa particularidad es muy relativa, dado que, en el interior del país la industria nacional es también, en gran medida, incapaz de suministrar a la agricultura - desde luego a la agricultura modernizada que es la que los demanda - los insumos de alto rendimiento que necesita.

Si bien el estado no fue muy eficaz en materia agrícola - al igual que en otras zonas de agricultura comercial - para nacionalizar la producción, la construcción y mantenimiento de la infraestructura de riego corrió a cuenta de la inversión pública federal y del financiamiento oficial. ${ }^{10}$

\footnotetext{
${ }^{9}$ En 1937, se establece por decreto presidencial la Zona Libre de Baja California, que incorpora el municipio de Mexicali y de Tecate, a los privilegios que ya gozaban los de Tijuana y Ensenada con el decreto de Perímetros Libres de 1933. Esta medida del régimen de Cárdenas es considerad a como una compensación por el asalto a las tierras. En el régimen de Zona Libre: “ . . a) no existen controles ni restricciones para importar, excepto en los casos (...) de mercancías que se produzcan, elaboren o transformen en la misma y b) los productos que entran a la zona no pagan ningún tipo de arancel (. . .) Sin embargo, a medida que se modificaban las condiciones socioeconómicas de la región, se fueron estableciendo controles y aranceles a la importación de determinados artículos, sobre todo para proteger a la industria local y favorecer e impulsar la integración económica con el resto del mercado nacional, en aquellos casos en que el suministro se hacía a precios competitivos". ZAZUETA, C., op. cit., pp. 62.63.

${ }^{10}$ En 1950 entró en operación la Presa Morelos, en 1955-58 se construyeron 630 pozos profundos: en 1965 se construyó el canal de desvío de las aguas salinas del Río Colorado, en 1964-72 se realizaron obras de rehabilitación de las tierras, canales, tomas de agua, nivelación de terrenos.
} 
Ya aquí hay notorias particularidades del valle con respecto a otras zonas de riego. La Presa Morelos es sólo derivadora del agua que el gobierno norteamericano le envía a través de sus presas de almacenamiento y obras de control del agua del río Colorado; no tiene capacidad de almacenamiento, ni de control del agua cuando los volúmenes exceden a los programados. La Representación Estatal de la Secretaría de Agricultura y Recursos Hidráulicos tiene que solicitar a las autoridades norteamericanas le envien, de la cuota anual que le corresponde a México, determinadas cantidades de agua en determinadas fechas del año, con base en una previa programación de los cultivos.

En la década de los sesentas aparece en el valle otro problema derivado de su situación fronteriza, de su contigüidad con territorio estadounidense: el alto grado de contaminación salina de sus tierras por las aguas del río Colorado. ${ }^{11}$ Esto provocó, entre otras cosas, la disminución alarmante de la fertilidad de los suelos al grado que, en 1964, la Secretaría de Agricultura y Ganadería estimó la pérdida del 30\% de la producción algodonera. Sumado a esto, a mediados de la década aparecen - por primera vez - fuertes problemas de plagas y enfermedades en el algodón. ${ }^{12}$

Sin embargo, aunque la superficie de algodón se reduce en un $9.8 \%$ de 1960 a 1966, la producción crece en un $35.6 \%$, denotando notables incrementos en los rendimientos por hectáreas (53.65\%). Es decir, en los años que preceden a la crisis, a pesar de los problemas de la salinidad ${ }^{13} y$ de las plagas, se observa un notable incremento de la producción y de los rendimientos del algodón, incrementos que parecen deberse a un uso más intensivo de insumos.

Aunque ambos problemas tuvieron efectos serios y dramáticos,,${ }^{14} \mathrm{con}$ sideramos que no es exacto que éstos hayan sido la causa determinante de la crisis. Al igual que en la agricultura del pais, en este período el crecimiento de la producción algodonera termina hacia los últimos años de los sesentas, con la baja estrepitosa de los volúmenes producidos y exportados. Esto

\footnotetext{
${ }^{11}$ En 1961, el gobierno norteamericano puso en operación 62 pozos profundos del valle del Welton. Mohawk, de los cuales se extraen aguas con alta concentración salina - como una operación de lavado de sus mantos acuíferos- que se incorporan a los caudales del río Colorado destinados al valle de Mexicali.

12 El sistema de monocultivo de cualquier planta - por ausencia de una rotación adecuada - conlleva a la proliferación de plagas y enfermedades y a mermar la fertilidad del suelo.

${ }^{13}$ Los años de 1961-65 fueron los más álgidos del problema de salinidad. "En 1965 el gobierno de Díaz Ordaz (. . .) construyó el canal de desvio que colocó a México en posición material y legal de desechar todas las aguas saladas del Welton-Mohawk, tirándolas al Golfo de California para no contaminar las aguas de riego del valle de Mexicali. Pero esta posición fue desechada. . ." López Zamora, E., op. cit., p. 93. Además, en 1964 el gohierno mexicano inicia la rehabilitación masiva de las tierras ensalitradas lo que, aunado a la no utilización del canal de desvio, denota que el problema fue controlado.

"4 En el invierno de 1961, ". . . una vez que se localizaron las fuentes de la contaminación (...) se iniciaron (en Mexicali) las manifestaciones de masas a las que concurrieron no sólo campesinos, xino personas de todos los estratos sociales, para protestar contra los actos del gobierno de Estados Unidos". Ibid., p. 70.
} 
sucede tanto con la producción nacional del trigo, incluso con el maíz y el frijol que, aunque su producción se orienta a satisfacer la demanda interna - más que los dos últimos que en el primero-, generaban importantes excedentes exportables; como con el henequén de Yucatán y el algodón del valle, cuyas fibras naturales no pudieron competir con las fibras sintéticas y fueron prácticamente sustituidos en el mercado internacional.

Esta crisis general se debió a las desventajas absolutas de nuestra producción en el mercado internacional. La caída de los precios y la reducción de la demanda del algodón del valle se deben, en primer término, a la competencia claramente ventajosa de las fibras sintéticas y, además, al incremento de sus costos de producción por los problemas de la salinidad y las plagas, que reduce aún más sus posibilidades de competir en el mercado mundial.

Asi, la causa determinante de la crisis del algodón del valle de Mexicali fue un problema de precios y de costos; fue la forma de inserción de la producción agrícola del valle en la economía mundial, su estrecha dependencia de un mercado en cuyos precios y demanda no influía mucho, y menos aún controlaba.

\section{PERIODO III: 1967/70-1986. INVERSION EXTRANJERA MEDIANTE EL SISTEMA DE AGRICULTURA POR CONTRATO}

Debido a la crisis del algodón hay un repliegue del capital norteamericano en el valle. Esto abandona el financiamiento indirecto del cultivo del algodón al no serle atractivo a partir de la caída de sus precios internacionales. Ante esta situación, se inicia una importante diversificación en el patrón de cultivos, en la que el Estado interviene canalizando financiamiento al algodón - cuyo cultivo representa una tercera o cuarta parte respecto al período anterior - y a otros cultivos, de los cuales algunos como el trigo tenían ya cierta presencia, y los forrajes y oleaginosas que prácticamente se inician.

Con la crisis de la producción agrícola del país en el mercado internacional, el capital extranjero adopta nuevas formas al disminuir su interés de invertir en la producción para el mercado externo y adquirir principal importancia en la producción para el mercado interno. Ahora, bajo la forma de inversión extranjera directa en la rama agroindustrial, ${ }^{15}$ elabora bienes alimentarios con alto valor agregado y bajo valor nutri-

\footnotetext{
15 De la reconversión económica de los países centrales en la década de los setentas -división internacional de la producción en base a costos de producción competitivos a escala internacional-. “. . se derivan modificaciones profundas en la forma de dependencia internacional. Nuevas formas de dependencia sobre todo industriales vienen a sustituir a las formas tradicionales agromercantiles. .." VERGOPOULUS, K. "La agricultura periférica en el nuevo orden internacional", periódico El Dia, suplemento "El gallo ilustrado", México, D.F., 1980.
} 
cional orientados al consumo masivo; paralelamente, incide en la producción pecuaria impulsando la aplicación de "paquetes tecnológicos" para la ganadería.

Con esto último, crece rápidamente el área de los cultivos destinados a forrajes y a la elaboración de alimentos balanceados, a la vez que se intensifica la producción pecuaria lo hace también la producción agrícola de cultivos "ganaderos". Esto permite que aumente la oferta de bienes pecuarios (carnes y lácteos) para estratos de altos ingresos. ${ }^{16}$

En este contexto y con el déficit de la producción agrícola nacional, el Estado intenta conformar un sistema alimentario nacional, integrando en mayor medida al sector agrícola con el resto de la economía, perdiendo importancia la orientación exportadora que tiene en el período anterior - al reorientarse la producción de las zonas agrícolas modernas hacia el mercado interno. ${ }^{17}$

Del mismo modo, la producción agrícola del valle se orienta hacia el mercado interno y se diversifica. ${ }^{18}$ Se observa el crecimiento en un grano básico para la alimentación de la población en los cultivos ganaderos y en la producción pecuaria destinados al mercado interno, principalmente local.

Desde luego, esa reorientación de la producción agrícola no satisface las expectativas de los agricultores del valle. ${ }^{19} \mathrm{Sin}$ embargo, con la coyuntura de los problemas laborales del "agribusiness" en el sur de California, a partir de 1970 y sobre todo en $1975 / 76$ aumentan las posibilidades de una nueva alternativa: el cultivo de hortalizas mediante contratos de producción.

En la década de los setentas, el auge del agronegocio en México se encuentra ". . . vinculado a la agricultura mediante sistemas de control de la producción agropecuaria, de los cuales el sistema de contratos es el

\footnotetext{
${ }^{16} \mathrm{Al} \mathrm{mismo} \mathrm{tiempo} \mathrm{se} \mathrm{produce} \mathrm{ganado} \mathrm{de} \mathrm{engorda} \mathrm{y} \mathrm{carne} \mathrm{magra} \mathrm{para} \mathrm{exportación,} \mathrm{que} \mathrm{el} \mathrm{mercado}$ norteamericano demanda.

${ }_{17}$ Las empresas transnacionales, en coinversión directa mayoritaria, son integrantes.integradoras de ese sistema a limentario en la medida en que existe realmente; controlan la actividad agrícola a partir de la etapa de industrialización y no per se, sino en la medida de crear una oferta de materias primas adecuada a sus necesidades. Es decir, en la tendencia a la integración vertical de estos complejos agroindustriales, el interés primordia) son lav etapas de mayor valor agregado (industrialización $y$ comereialización); la producción agrícola interesa sólo para asegurar el abastecimiento.

18 El trigo es el más importante en cuanto a superficie, su producción se destina casi totalmente al mercado local, así también el zacate rye grass y la alfalfa; el sorgo y la cebada se destinan a pequeñas industrias de la localidad, una para alimentos balanceados y la otra para malta cervecera; la superficie de algodón se reduce a una cuarta parte de la que tenía en el periodo anterior y su producción se destina al mercado interno cuando las condiciones en el mercado internacional son muy desfavorables.

${ }^{19}$ Según CEPAL, Economía campesina y agricultura empresarial, Ed. Siglo XXI, México, 1982. En el estado de Baja California en 1970, el $63 \%$ de los ejidatarios y el $65 \%$ de los propietarios son agricultores medianos y ("transicionales") empresarios, es decir, productores no campesinos. Y, considerando que el $100 \%$ de la tierra cultivada en el valle de Mexicali es de riego, lo que representa el 95.5\% de la superficie de riego en la entidad, esos porcentajes muy probablemente son más elevados entre los productores agrícolas del valle en particular.
} 
más extendido [. . .] Este supone que las empresas suministran créditos, asistencia técnica, insumos y hasta maquinaria agrícola a cambio de un compromiso, en ocasiones escrito, de entrega de la cosecha por parte del productor. ${ }^{20}$

Sin embargo, todo este panorama de reorganización no representa una solución al problema de la crisis. Con la caída de los precios del petróleo, el enorme endeudamiento externo y la devaluación acelerada a partir de los primeros años de la presente década, manifiestan con toda su crudeza las "desventajas absolutas" 21 de la producción nacional. La tendencia a los bajos precios y altos costos, la ausencia de capacidad competitiva - salvo en algunos cultivos en que tenemos algunas ventajas de clima, de geografía y, sobre todo, de precio de la fuerza de trabajo-, parecen irreversibles dentro de la nueva situación de nuestra producción en el mercado internacional.

En este contexto, el cultivo de hortalizas es una alternativa limitada en cuanto a los alcances que podría tener para dinamizar el resto de la agricultura del valle. Representa sólo alrededor del $\mathbf{4} \%$ de la superficie cultivada y aproximadamente el $1.5 \%$ de los productores; sin embargo, resulta significativa: genera aproximadamente el $25 \%$ del valor de la producción agrícola y el $29 \%$ de las jornadas/hombre. El capital extranjero sigue teniendo presencia, aunque más reducida en espacio, más directa y localizada.

La agricultura por contrato presenta diversas caracteristicas en función de la diversidad de condiciones y propósitos en que se utiliza. En el caso de las hortalizas, el propósito fundamental de los inversionistas norteamericanos es mantenerse en el mercado estadounidense durante todo el año, incluyendo los meses en que su propia oferta resulta insuficiente. En este sentido, la oferta de hortalizas de Sinaloa complementa la de Florida y la del valle - junto con la Ensenada- de California, ${ }^{22}$

\footnotetext{
20 RAMA, Ruth y Rello, Fernando, "La internacionalización de la agricultura mexicana", Mimeo, "Coloquio sobre agricultura mexicana", UNAM. México, 1979. p. 10.

21 Desde fines de los sesentas, con las enormes fluctuaciones de la produceión agrícola de esos años, el Estado anunció la política de las llamadas "ventajas comparativas"; se intentaba producir sólo aquello en lo que tenemos ventajas con respecto de otros países, y comprar en el exterior aquello en lo que tenemos desventajas. El llamado "modelo de desarrollo compartido" del régimen echeverrista. con sus enormes flujos financieros al campo - recursos obtenidos mediante endeudamiento externo-, constituyó un paliativo que impidió que se hiciera del todo efectiva tal intención. En el siguiente sexenio, con el crecimiento sin precedentes de las exportaciones petroleras, se hizo posible esa política al intercambiarse petróleo por alimentos; política que ha debido llamarse de las "desventajas absolutas" ya que se trata de producir o dejar de producir en función de la cumpetitividad de nuestros costos medios internacionales.

22 La producción del valle en algunas hortalizas tiene una desventaja: al formar una unidad físicaclimatológica con el Valle Imperial sus cielos coinciden, tiene una produccín cuando en el mercado norteamericano abunda y el gobierno estadounidense incrementa enormemente los aranceles de importación.
} 
aunque hay una gran diferencia en la proporción en que ambas entidades (Sinaloa y Baja California) lo hacen. ${ }^{23}$

Es en la década de los setentas cuando se inicia el crecimiento de los cultivos de hortalizas en el valle ${ }^{24}$ debido, por un lado, a la reducción forzosa de la superficie del algodón; y, por otro, al mayor interés que agricultores y comerciantes norteamericanos mostraron en desplazar parte de la producción del sur de California, por los conflictos laborales con los trabajadores agrícolas que allí tuvieron. Se establecieron contratos para cultivar melón, cebollín, lechuga, calabacita, rábano, brócoli y coliflor.

Los antecedentes de la inversión extranjera en el valle mediante contratos de producción, se encuentran desde 1957 con el cultivo del espárrago. En 1955, la disminución de la superficie del algodón derivada de la reducción de los volúmenes de agua del río Colorado, y con la ayuda del 'dumping' que Estados Unidos provocó en el mercado internacional del algodón, contribuyó a que se diera cierta diversificación en los cultivos y cierto impulso a las hortalizas como una alternativa para aprovechar el agua disponible en cultivos de alto rendimiento; $y$, por primera vez desde 1937, genera volúmenes para su comercialización.

Es entonces que se inicia el cultivo de espárrago. Su producción se realiza totalmente mediante contratos de producción y está destinada completamente al mercado norteamericano. Pero sólo hasta los años setentas, cuando se inicia el crecimiento del resto de las hortalizas, es que se amplía su superficie y se incrementa su producción.

La producción de hortalizas en el valle para el mercado norteamericano, empezó a crecer a partir de 1971; tuvo un crecimiento importante en 1975 que se desaceleró hacia 1982 y, desde entonces con el impulso de la devaluación ha tenido un crecimiento paulatino sostenido. Aunque está imposibilitada para convertirse en complemento de la producción de California en los períodos del año en que Estados Unidos no tiene oferta suficiente, y ésta es la ventaja de las hortalizas de Sinaloa; sus posibilidades de un mayor crecimiento estriban en factores que permitan un mayor desplazamiento de la producción de California, a partir de los cuales tendría la ventaja de la distancia respecto de otras zonas agrícolas fuera de territorio estadounidense. Tales factores serían situaciones de la horticultura de California, en la que las ventajas potenciales del valle - tales como el precio de la fuerza de trabajo, el precio del suclo, el margen en cuanto a problemas de contaminación-, tuvieran un peso suficiente que las convirtieran en ventajas reales.

${ }^{23}$ Según la Unión Nacional de Productores de Hortalizas, en la temporada 1980-81, Sinaloa contribuyó con el $58.7 \%$ y Baja California con el $6.3 \%$ del volumen total exportado.

${ }_{24}$ En los origenes del valle, los chinos introdujerun el cultivo de hortalizas en pequeña escala para consumo familiar y para comercializar, ellos mismos los excedentes en el mercado local. En 1937 con la expropiación de las tierras, los chinos se retiran de la agricultura y emigran a la ciudad de Mexicali donde se dedican al comercio y los servicios urbanos. Esto y la situación enormemente favorable del algodón en el mercado mundial hícieron que el cultivo de hortalizas perdiera la relativa importancia que habia tenido en el período anterior. 


\section{BIBLIOGRAFIA}

CEPAL. Economia campesina y agricultura empresarial. Ed. Siglo XXI, México. 1982.

LOPEZ Zamora, Emilio. El agua, la tierra, los hombres de México. Ed. FCE, México. 1977.

RAMA, Ruth y Rello, Fernando. La Internacionalización de la agricultura mexicana. Mimeo. Coloquio sobre Agricultura Mexicana. UNAM, México, 1979.

VERGOPOULUS, Kostas. La Agricultura periférica en el nuevo orden internacional. Perí́dico El Día, suplemento "El gallo ilustrado", México, 1980.

ZAZUETA, Carlos. La formación de la frontera norte: El caso de Baja California. Tesis de maestría, El Colegio de México, México. 1978. 